\title{
Management of Acute Facial Paralysis
}

\author{
Raghu Nandhan Sampath Kumar PhD, FRCS (ORL-HNS) \\ Richard M. Irving MD, FRCS (ORL-HNS)
}

\section{INTRODUCTION}

Face is the window to the mind and facial expressions reflect the emotions and wellbeing of the person. Hence, acute facial weakness is probably the most visible and distressing nerve palsy in the body, since it manifests with cosmetic deformity along with functional problems of eyelid closure, oral continence and speech. The prevalence of acute facial palsy in the general population of the United Kingdom (UK) is about 1:5000, making it a common clinical entity which comes through the doors of emergency medicine and for all general practitioners to be familiar with. ${ }^{1}$ A systematic approach to the correct diagnosis for facial weakness and timely appropriate management is vital in providing the best chance for its complete recovery.

The facial nerve is a mixed nerve containing motor, sensory and parasympathetic fibres however, it is the motor component that predominates and produces the greatest morbidity associated with conditions affecting the nerve. Each facial nerve has approximately 10,000 fibres of which two thirds are motor and the remaining one third are sensory. ${ }^{1}$ It is the commonest cranial nerve to be involved in functional deficit due to its long and tortuous course (Figure 1). It commences from the brainstem running intra-cranially along the cerebello-pontine angle and traverses a non-expandable bony canal within the petrous temporal bone where it takes 2 acute bends (genus) through the middle ear cleft and exits through the stylomastoid foramen to traverse through the parotid gland to supply the hemi-facial muscles. ${ }^{1}$ This complex anatomy may account for its vulnerability to involvement by trauma, inflammation or neoplasia.

Embryologically, facial nerve is derived from the second branchial arch (Hyoid arch). It develops during the time which closely adjacent derivatives of the first arch give rise to the external and middle ear components, hence anomalies of the facial nerve are more common where malformations of the external or middle ear are present. ${ }^{2}$ In children the facial nerve approximates to that found in adults, except that it exits through the more superficially located stylomastoid foramen with ongoing development of the mastoid tip as a traction epiphysis from the time of birth. Hence, at birth there is a possibility of acute facial palsy due to forceps delivery and there is also a higher risk of facial nerve injury in children due to surgery or trauma at this point of exit from the temporal bone. ${ }^{2}$

The facial nerve comprises of both upper and lower motor neurons. Upper motor neuron (UMN) weakness occurs due to lesions involving the motor cortex or internal capsule, while lower motor neuron (LMN) weakness is due to affliction of the nerve in its intracranial, intratemporal and extracranial segments. Pathognomonically upper motor neuron palsy spares the forehead due to bilateral cortico-bulbar innervation that results in weakness of the contralateral lower facial muscles only, while lower motor neuron weakness involves the entire ipsilateral hemi-facial muscles. ${ }^{1}$

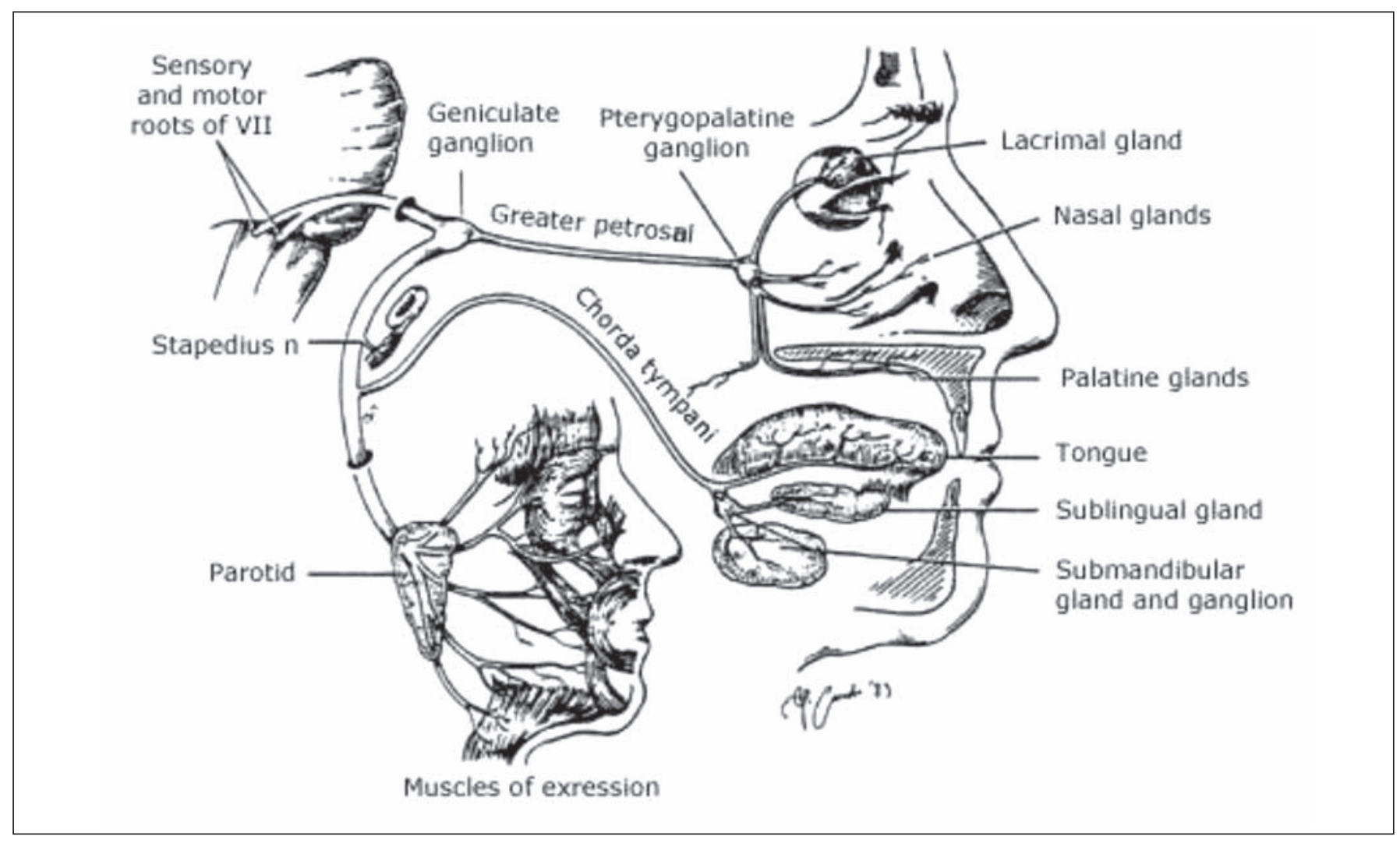

Figure 1: Anatomical course \& supply of the facial nerve 
Clinical judgement of the site of facial nerve injury is known by evaluating for abnormal loudness of sounds (noted in loss of stapedius function), change in taste in ipsilateral anterior two-thirds of tongue (chorda tympani involvement) and anaesthesia in postero-superior canal wall skin (Hitselberger's sign seen in acoustic neuroma, $10 \%$ of which can present with acute facial weakness). The labyrinthine segment is the narrowest segment of the facial nerve canal and therefore susceptible to compression secondary to inflammation and to vascular insults, as it lacks epineurium and anastomosing arterial cascades. Idiopathic (Bell's palsy) is most commonly due to oedema at this segment. In such cases loss of tears and saliva may be features presenting along with facial palsy. Bell's palsy patients may also perceive pain around the stylomastoid foramen as the nerve is inflamed at this exit point. ${ }^{1}$

Variations in the anatomical course of the facial nerve may exist both in its intra-temporal and extra-cranial segments. The fallopian canal in the middle ear cleft may

\begin{tabular}{|l|l|}
\hline Idiopathic & Bell's palsy \\
\hline Infection & Acute or chronic otitis media \\
& Cholesteatoma \\
& Malignant otitis externa \\
Skull-base osteomyelitis \\
Lyme disease \\
Syphilis \\
Herpes simplex \\
Varicella zoster \\
Ramsay-Hunt syndrome
\end{tabular}

Table 1: Differential diagnoses of acute facial nerve palsy be dehiscent in up to $50 \%$ of cases especially if congenital ear malformations co-exist. Facial palsy occurs in about $6 \%$ of acute otitis media, where this segment is involved. Variation may exist in the final divisions after exiting the stylomastoid foramen. ${ }^{1}$ Hence it is important to check muscles supplied by each branch individually to arrive at a grade for the facial weakness correctly.

\section{AETIOLOGY OF FACIAL PALSY}

Acute facial nerve paralysis can result from numerous causes, as listed below (Table 1) the most common being idiopathic or Bell's palsy, trauma, infection and tumours. ${ }^{3}$

\section{EVALUATION OF FACIAL WEAKNESS}

History is paramount in identifying the aetiology of facial weakness. A thorough history regarding onset, duration and progress of weakness, past and preceding otological history, presence of coexistent neurological symptoms and systemic illnesses would help the clinician to arrive at a diagnosis from the differentials quoted above. ${ }^{4}$ It is vital to perform a comprehensive ENT and head and neck examination including otoscopy to identify obvious pathology leading onto the facial palsy. Examination is incomplete without testing all cranial nerves and assessment of hearing and balance functions. Systemic examination should include checking for diabetes and hypertension.

Bell's palsy is a diagnosis of exclusion of other causes and should not be the provisional diagnosis for commencing treatment without confirmation. Red flags which warn against the diagnosis of Bell's palsy are obvious otological symptoms and the presence of bilateral facial palsy, slurred speech, hemiparesis/paraesthesia of the face and limbs, cerebellar signs, multiple cranial neuropathy, previous history of facial weakness or history of locoregional cancers. ${ }^{4}$

Recurrent or bilateral palsy warrants an urgent high resolution CT/MRI scan to look for neurological causes. ${ }^{5}$ All trauma cases require CT scan of brain and petrous temporal bones as top priority to assess the site of nerve injury. Immediate post-traumatic total facial palsy indicates obvious trauma to the nerve, while delayed onset facial weakness in the background of recent head injury suggests oedema of the nerve which is possibly in anatomical continuity. ${ }^{4}$ Urgent ENT referral is important in both scenarios and audiograms are indicated in all temporal bone trauma. Blood tests should be targeted at screening for infective sources such as Syphilis, Lyme's disease, HIV, $\mathrm{TB}$ and EBV. If infection is ruled out auto-immune screen and connective tissue screen is recommended. ${ }^{3}$

Facial palsy should always be graded at first and all subsequent consultations. Facial grading scales are used in measuring recovery, potential deterioration and comparison of facial nerve outcomes across therapeutic modalities. The House-Brackmann (HB) score (Table 2), first described in 1985 is the most popular and widely used clinical measure of the degree of facial motor weakness. It comprises of 6 grades from normal (HB I) to total paralysis (HB VI) ${ }^{6}$ 


\begin{tabular}{|l|l|l|l|l|l|}
\hline Grade & Gross & Resting Tone & Forehead & Eye Closure & Mouth \\
\hline I & \multicolumn{5}{|c|}{ Normal symmetrical function in all areas } \\
\hline II & Slight weakness & Normal & Moderate- good function & $\begin{array}{l}\text { Complete closure } \\
\text { with minimal effort }\end{array}$ & Slight asymmetry \\
\hline III & Obvious weakness & Normal & Slight-moderate function & $\begin{array}{l}\text { Complete closure } \\
\text { with full effort }\end{array}$ & Slight weakness \\
\hline IV & Disfiguring & Normal & None & Incomplete closure & Asymmetric \\
\hline V & $\begin{array}{l}\text { Barely perceptible } \\
\text { motion }\end{array}$ & Asymmetric & None & Incomplete closure & Slight movement \\
\hline VI & \multicolumn{5}{|c|}{$\begin{array}{l}\text { Total Paralysis } \\
\text { (No movement, loss of tone, no synkinesis, contracture or spasm) }\end{array}$} \\
\hline
\end{tabular}

Table 2: House-Brackmann Grading

Management protocols for common conditions presenting with acute facial paralysis are briefly discussed below:

\section{Bell's Palsy}

Described first by Sir Charles Bell in 1821, this is an idiopathic unilateral peripheral facial nerve palsy with some evidence to support a viral aetiology. Annual incidence is 20 to 30 cases per 100,000 people per year, with an equal sex incidence and a peak incidence of 40 years, although it can occur in all age groups. ${ }^{7}$ Patients who have had an episode of Bell's Palsy have an $8 \%$ risk of recurrence. Although Bell's palsy is the commonest cause of unilateral facial paralysis, accounting for $60-70 \%$ of all cases, it is a diagnosis of exclusion. The majority of patients will show partial recovery within 3 weeks of onset and all patients get some recovery. Poor prognostic factors include complete facial palsy at onset, age over 60 years, severe pain, no recovery by 3 weeks, and associated conditions e.g. hypertension and diabetes. ${ }^{8}$

Bell's palsy normally presents with sudden onset of unilateral lower motor nerve facial palsy over a course of 24-48 hours. It is frequently preceded by periauricular parathesia or otalgia. A viral prodome may exist and dysgeusia, hyeracusis and facial numbness may be present. Tearing mechanism is affected but paradoxically the patient may complain of excess tears due to loss of lower lid control. ${ }^{7}$ Examination includes assessment of facial nerve function, remaining cranial nerves, otoscopy and palpation of the parotid. Specifically the degree of eye closure and presence of Bell's phenomenon should be determined to quantify corneal risk. A baseline pure tone audiogram should be obtained, especially if the patient complains of hearing loss, as this is not typical in Bell's Palsy. Ocular care takes precedence in cases where there is incomplete eye closure, to prevent sight threatening complications. ${ }^{7}$ Urgent referral to ENT and Ophthalmology should be considered in all cases of total facial paralysis (Figure 2).

\section{Treatment Algorithm}

1. Eye care - Hypromellose eye drops during the day/ Lacrilube ointment and eye tape at night if incomplete eye closure (above Gr-III palsy).
2. Antibiotics - (only if suspected otitis media/URTI) Amoxicillin orally $15-20 \mathrm{mg} / \mathrm{kg}(\max 500 \mathrm{mg}) 3$ times a day for 14 days. If penicillin allergy $(<12$ years old $)-$ Azithromycin $10 \mathrm{mg} / \mathrm{kg}$ (max $500 \mathrm{mg}$ ) once a day for 3 consecutive days, then repeated for 3 consecutive days in week 2 . If penicillin allergy (> 12 years old) - Doxycycline $100 \mathrm{mg}$ twice daily for 14 days.

3. Steroids - Prednisolone $1 \mathrm{mg} / \mathrm{kg}$ (60mg maximum) for 5-7 days if patients presents within 72 hours of symptom onset (no need to wean). PPI needs to be added with oral steroids. Literature shows that corticosteroids are 'the drug of choice' in treatment of Bell's Palsy. ${ }^{8}$

4. Antivirals - Cochrane review suggests 'no significant benefit' of antivirals compared with placebo in producing complete recovery from Bell's palsy. Literature also shows that antivirals are significantly less likely than corticosteroids to produce complete recovery.

5. Recommended urgent referrals: ENT - if unsure of diagnosis. All adults with associated red flags of ENT/H head and neck pathology and all children with facial palsy need referral to ENT.

Ophthalmology - indicated if eye is not fully closing at night (above Gr-III).

Neurology - if focal or evolving neurological signs, UMN or bilateral facial weakness and multiple cranial nerve palsy.

Physiotherapy - if weakness is still present at 6 weeks post-treatment follow up (nerve conduction study recommended)

Audiology/Speech and Language Therapy - if difficulties with hearing, speech \& swallowing.

Outcome at follow up depends upon the factors of age, aetiology, degree of palsy at first presentation, duration of weakness, presence of medical comorbidities/ systemic illnesses and compliance to treatment. Prognosis is expected to be good with complete resolution of weakness within 4 weeks in the majority of patients $(>85 \%)$ with Bell's palsy. ${ }^{7}$ If no recovery is felt by 3 months, an alternative diagnoses must be considered with appropriate investigations in a specialist facial palsy clinic for further management. The options for facial reanimation are aimed at improving cosmesis and restoring function. This may include facial physiotherapy, Botox injections and surgical reanimation techniques performed under the joint care of expert otologists and facial plastic surgeons. 


\section{Otologic Infections}

Bacterial causes for facial nerve paralysis include acute or chronic otitis media and skull base osteomyelitis. Facial paralysis secondary to acute otitis media is rare and tends to be more common in young children. Middle ear cholesteatoma is associated with facial paralysis in under $1 \%$ of cases however when the disease is in the petrous apex this increases to close to $50 \% .^{9}$ Most cases of facial paralysis secondary to acute otitis media resolve with conservative management in the form of systemic antibiotics. Surgical options of myringotomy with or without ventilation tube may be indicated when spontaneous perforation of the tympanic membrane is not present.

A mastoidectomy may be indicated for suppurative complications, lack of clinical improvement or worsening of the facial palsy. Rarely, surgical decompression of the facial nerve is necessary. The converse generally is true for chronic otitis media or cholesteatoma or the delayed onset of facial paralysis, as this is probably secondary to erosion of the facial nerve canal and early surgical intervention is warranted in the form of a mastoidectomy. Recovery can occur even if treatment is delayed for several months in facial paralysis secondary to cholesteatoma. ${ }^{?}$

Lyme disease and Ramsey-Hunt syndrome are uncommon infections causing acute facial palsy. Lyme disease is a multi-systemic illness caused by the Spirocheate Borrelia Burgdorferi and is transmitted by tic bites and can give rise to both unilateral or bilateral facial nerve paralysis. Erythema migrans is evident in the early stages of the disease with associated joint pain, fever and later symptoms of fatigue and neck stiffness. Enquiring about recent travel or outdoor activities such as hiking or camping is important to establish the aetiology. The diagnosis is confirmed by serology and treatment is with long term antibiotics. ${ }^{10}$

Ramsey-Hunt syndrome is caused by the varicella zoster virus and is characterised by vesicles in the external ear, soft palate or tongue. It may be misdiagnosed as Bell's palsy as the classic vesicles may not appear or may be delayed. Varicella zoster virus polymerase chain reaction (PCR) may help to distinguish between Ramsay Hunt syndrome and Bell's palsy patients. If the diagnosis is suspected, prompt treatment with steroids and antivirals is recommended. However despite treatment the prognosis is poor with fewer than half of patients achieving complete recovery of facial function. ${ }^{4}$

\section{Temporal Bone Trauma}

Both blunt and penetrating mechanisms of injury may result in acute facial nerve paralysis. Causes of injury include motor vehicle accidents, assault, stab and gunshot injuries and iatrogenic injury. Temporal bone fractures account for $18-22 \%$ of skull fractures in patients treated for head trauma. Common sequelae of temporal bone fractures include facial nerve injury, damage to the cochleovestibular apparatus with associated sensorineural hearing loss, conductive loss, balance disturbance, tinnitus, vertigo and cerebrospinal fluid leak. ${ }^{11}$

Traditional classification systems of longitudinal, transverse and mixed temporal bone fractures, are still used but poorly correlate to clinical complications (Figure 2a \& 2b). More recently, petrous or otic capsule sparing versus non-petrous or otic capsule violating classifications have been suggested to provide improved clinical correlation, aiding early recognition of potential complications and guiding management. ${ }^{11}$ Facial nerve injury is more common in transverse (30-50\%) compared to longitudinal (10-25\%) temporal bone fractures and less common in children. Gunshot related temporal bone injury results in extensive injury to adjacent structures, with a much higher incidence of facial nerve paralysis compared to closed head injury. As a result, these injuries are more likely to require surgical exploration than blunt temporal bone fractures. ${ }^{12}$

A complete neuro-otological examination of the patient is required, including; otoscopy for haemotympanum/perforated eardrum, evidence of postauricular ecchymosis (Battles sign), Cerebro Spinal Fluid leak from the ear or nose and in the conscious patient assessment of facial nerve function, nystagmus and hearing loss (bedside tuning fork test and formal audiometric testing at the earliest opportunity). In the critically ill patient, rapid imaging with high resolution $\mathrm{CT}$ is essential to evaluate the temporal bone but also the intracranial contents and potential cervical spine injury. ${ }^{11}$

In acute facial palsy post ear surgery, iatrogenic injury of the tympanic segment of the facial nerve is the most common site of injury, due to the prevalence of facial canal dehiscence or exposure secondary to cholesteatoma. The vertical segment is more prone to trauma during posterior tympanotomy for cochlear implantation or combined approach tympanoplasty. The nerve can also be damaged in the cerebellopontine angle during skull base neurosurgery or in its extra-temporal segment while operating on the parotid gland.

In general post-traumatic delayed onset or incomplete paresis mostly recover completely, the majority within the first 3 months. Treatment involves high dose corticosteroids and eye care. The decision to explore a facial nerve following trauma is complex. Factors favouring exploration and indicating a severe injury to the nerve are a penetrating injury, immediate onset of paralysis, CT evidence of nerve canal disruption or bony spicule, loss of inner ear function, persistent CSF leak and $90 \%$ or greater degeneration on electroneurography. Iatrogenic injury should be repaired at the time of surgery if noticed or re-explored within days and the nerve repaired. Patients with persistent facial paralysis are observed for at least a year before considering reanimation and reinnervation techniques, to allow for natural recovery. ${ }^{12}$

\section{Facial Nerve Tumours}

Tumours account for less than $5 \%$ of patients with facial nerve paralysis. These may present with acute, recurrent or progressive weakness. Schwannomas form the majority of intrinsic facial nerve tumours and can occur anywhere along the course of the facial nerve (Figure 3a \& 3b). ${ }^{3}$ Haemangiomas are extremely rare benign vascular tumours and tend to arise around the geniculate ganglion and internal auditory canal, reflective of the rich blood supply at these sites. ${ }^{13}$ Malignant involvement includes direct perineural spread via the facial nerve into the temporal bone by parotid mucoepidermoid and adenoid cystic carcinoma and squamous cell carcinoma. ${ }^{3}$ 


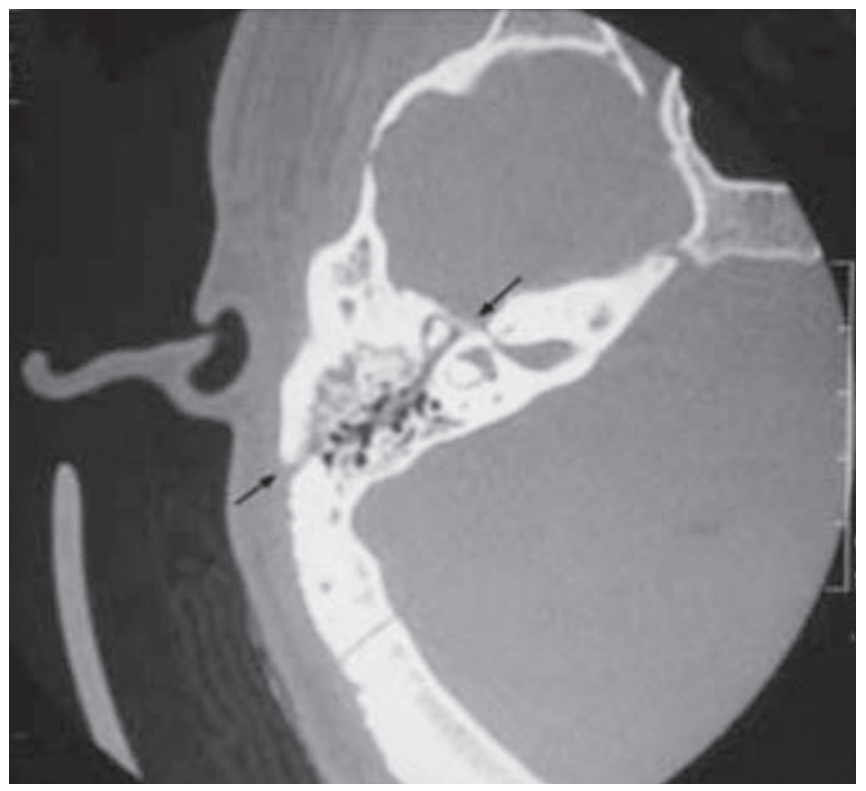

Figure 2a: Longitudinal fracture of temporal bone

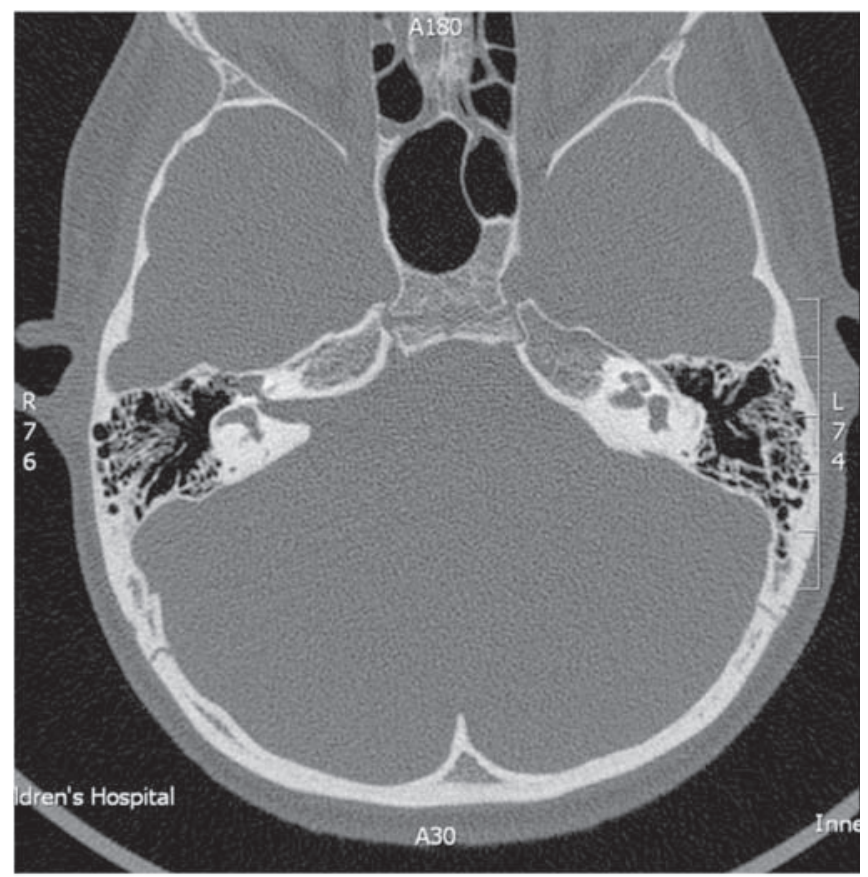

Figure 3a: Schwannoma of the right geniculate ganglion

Eliciting a past history of recurrent or progressive facial nerve paresis or absence of recovery after 3 months having been treated as Bell's palsy, and presence of facial spasms or twitching, are suggestive of a tumour. ${ }^{5}$ Hearing loss may be sensorineural with associated tinnitus or vertigo with tumours in the labyrinthine segment or conductive with tumours in the horizontal segment. Examination should include assessment of facial nerve function, otoscopy, palpation of parotid and neck. Thinsectioned contrast enhanced MRI in combination with high resolution CT of temporal bone is recommended. ${ }^{13}$ All such patients need urgent referral to Otologists for further management.

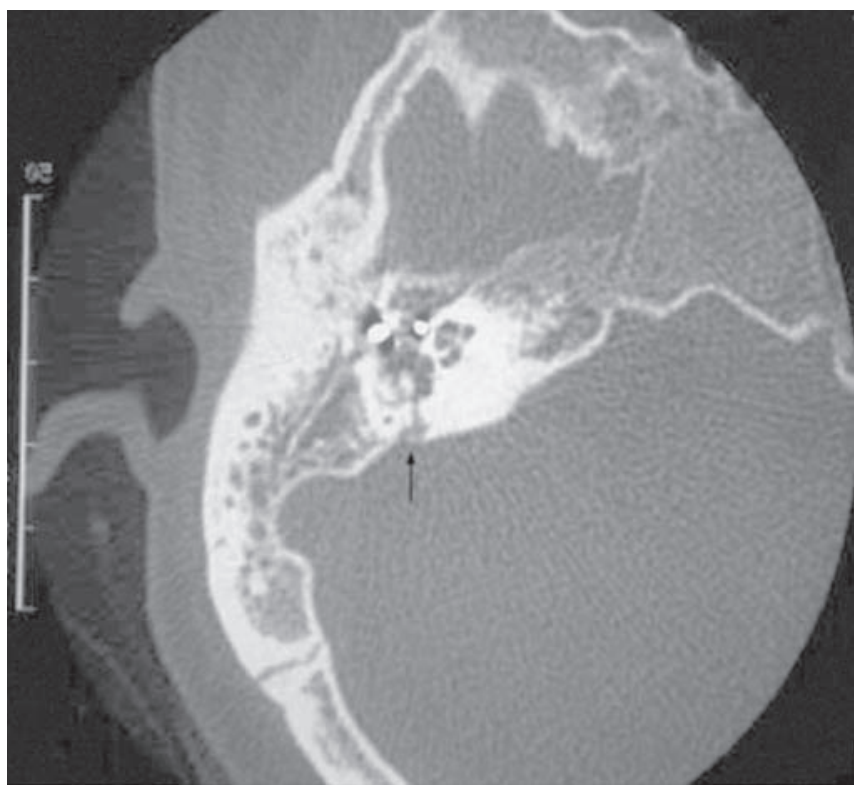

Figure 2b: Transverse fracture of temporal bone

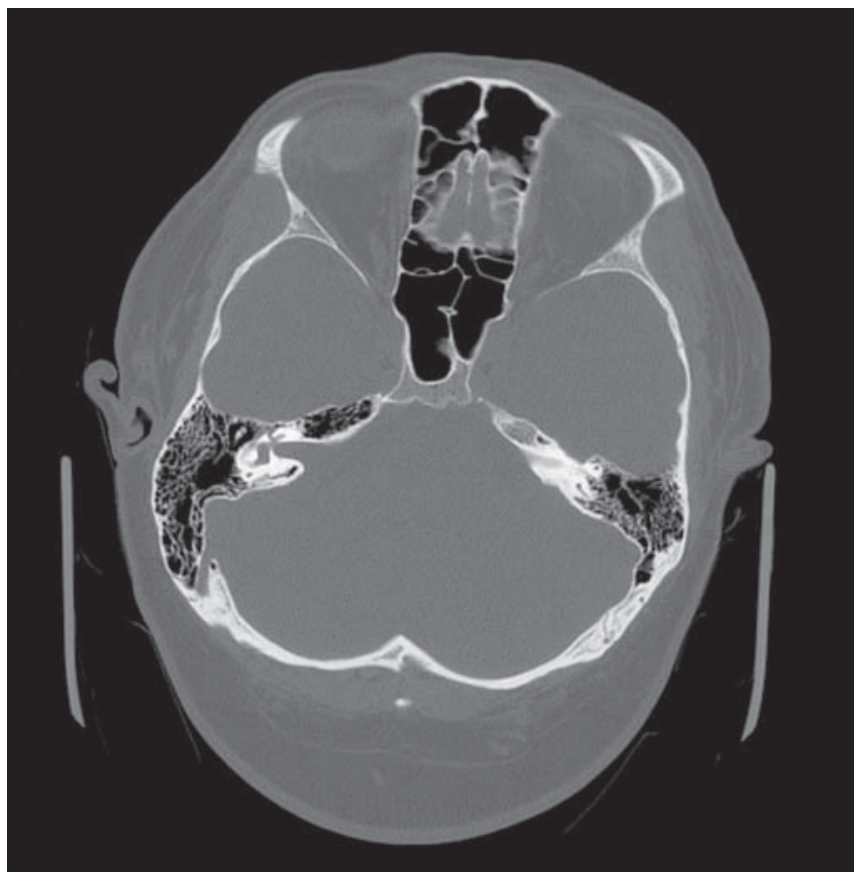

Figure 3b: Normal geniculate ganglion for comparison

\section{CONCLUSION}

Acute facial paralysis is a fairly common entity that every clinician will encounter during their practice. It is prudent to have a general concept of the facial nerve and follow a systematic approach to the clinical evaluation and appropriate management of these cases. If the diagnosis is in doubt or if treatment outcome is not satisfactory, urgent referral to an Otologist is strongly advised.

\section{REFERENCES}

1. Hohman, M. H. \& Hadlock, T. A. Etiology, diagnosis, 
and management of facial palsy: 2000 patients at a facial nerve center. Laryngoscope 2014; 124:E283-93.

2. Sataloff, R. T. \& Selber, J. C. Phylogeny and embryology of the facial nerve and related structures. Part II: embryology. Ear Nose Throat 7 2003;82(10): 764-6, 769-72, 774 .

3. May, M. \& Klein, S. R. Differential diagnosis of facial nerve palsy. Otolaryngologic Clinics of Nor Am 1991, vol. 24, no. 3, pp. 613-45.

4. Finsterer, J. Management of peripheral facial nerve palsy. Eur Arch Otorbinolaryngol 2008; 265:743-52.

5. Doshi, J. \& Irving, R. Recurrent facial nerve palsy: the role of surgery. F Laryngol Otol 2010; 124(10): 1202-4.

6. House, J. W. \& Brackmann, D. E. Facial nerve grading system. Otolaryngol Head Neck Surg.1985; 93:146-7.

7. Gilden, D. H. Clinical practice. Bell's Palsy. N Englf Med 2004; 351(13): 1323-31.

8. Axelsson, S., Berg, T. \& Jonsson, L. Prednisolone in Bell's Palsy related to treatment start and age. Otol Neurotol 2011; 32:141-6.

9. Siddiq, M. A., Hanu-Cenat, L. M. \& Irving, R. M.
Facial palsy secondary to cholesteatoma: analysis of outcome following surgery. 7 Laryngol Otol 2007; 121(2): 114-7.

10. Alaani, A., Hogg, R., Saravanappa, N. \& Irving, R. M. An analysis of the diagnostic delay in unilateral facial palsy. 7 Laryngol Otol 2005; 119(3): 184-8.

11. Johnson, F., Semaan, M. T. \& Megerian, C. A. Temporal bone fracture: evaluation and management in the modern era. Otolaryngol Clin N Am 2008; 41: 597-618.

12. Moore, P. L., Selby, G. \& Irving, R. M. Gunshot injuries to the temporal bone. 7 Laryngol Otol 2003; 117(1): 71-4.

13. Salib, R. J., Tziambazis, E., McDermott, A.L., Chavda, S. V. \& Irving, R. M. The crucial role of imaging in detection of facial nerve haemangiomas. $\mathcal{F}$ Laryngol Otol 2001; 115(6): 510-3.

Correspondence to: Dept of Neurotology and Skullbase Surgery, Queen Elizabeth University Hospitals, Birmingham, UK richard.irving@uhb.nhs.uk

\section{Skiing Knee Injury: The relevance of subtle radiographic findings}

O. Abdulla, M.J. Naqvi, R. Proctor FRCR, MRCP, MRCGP, MA(Cantab), S. Shamshuddin MBBS, DMRD, FRCR, Department of Radiology, Lancaster Royal Infirmary

\section{CLINICAL PRESENTATION}

A 24 year old lady sustained a knee injury whilst skiing and underwent plain knee radiographs. Subtle features were identified on the knee radiograph which warranted further imaging with MRI.

\section{IMAGES}

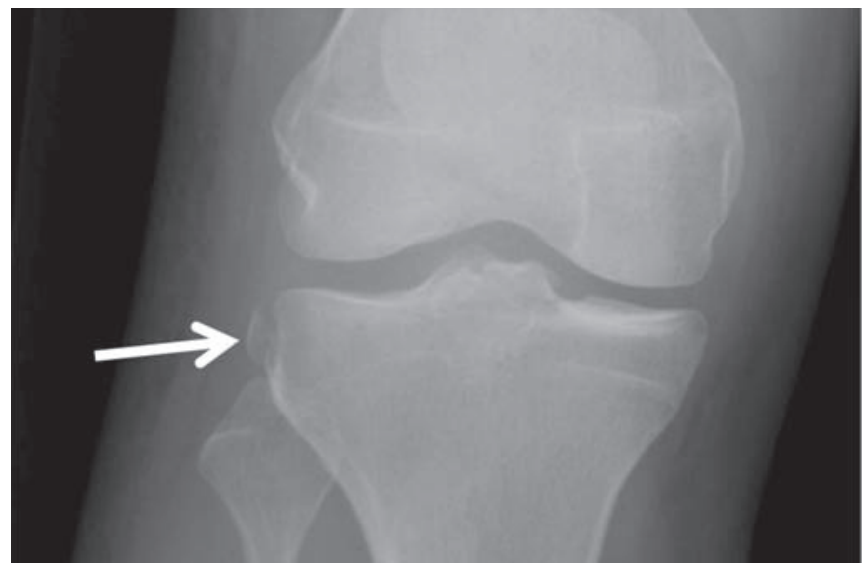

Figure 1a: AP knee radiograph shows subtle avulsion fracture from the lateral margin of the lateral tibial plateau, termed a Segond fracture (arrow)

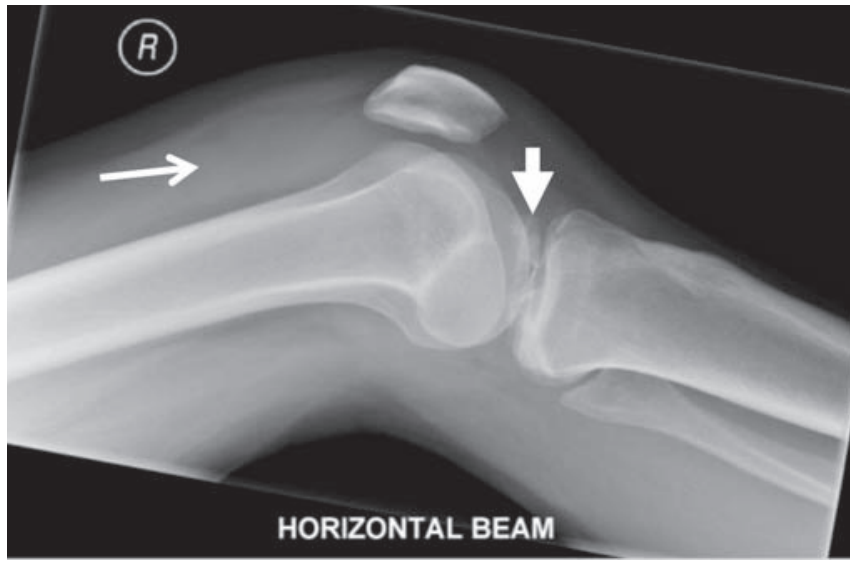

Figure 1b: Lateral knee radiograph shows an intra-articular bone fragment (short arrow) and moderate joint effusion (arrow) 